\title{
Charm and Beauty Production in Deep-inelastic Scattering at HERA
}

\section{Stefan Schmitt ${ }^{* \dagger}$}

DESY, Notkestr. 85, 22607 Hamburg, Germany

E-mail: stefan.schmitt@desy.de

\begin{abstract}
Measurements of open beauty and charm production cross sections in deep inelastic scattering (DIS) at HERA by the H1 and ZEUS Collaborations are combined. Reduced cross sections for beauty and charm production are obtained in $e p$ collisions at a centre-of-mass energy $\sqrt{s}=319 \mathrm{GeV}$ in a kinematic range of the photon virtuality $2.5<Q^{2}<2000 \mathrm{GeV}^{2}$ and the Bjorken scaling variable $3 \times 10^{-5}<x<5 \times 10^{-2}$. The combination method accounts for the correlations of the systematic uncertainties among the different data sets. The combined data are compared to perturbative QCD predictions at next-to-leading order (NLO). The running charm and beauty quark masses are extracted. Furthermore, open charm production is studied in diffractive DIS at $5<Q^{2}<100 \mathrm{GeV}^{2}$, based on data recorded by the $\mathrm{H} 1$ experiment. The event topology is given by $e p \rightarrow e X Y$, where the system $X$, containing at least one $D^{*}(2010)$ meson, is separated from a leading momentum low-mass proton-dissociative system $Y$ by a large rapidity gap. The measured cross sections are compared with NLO QCD predictions based on previously determined diffractive parton densities of the proton.
\end{abstract}

The European Physical Society Conference on High Energy Physics

5-12 July, 2017

Venice

${ }^{*}$ Speaker.
${ }^{\dagger}$ For the $\mathrm{H} 1$ and ZEUS collaborations 


\section{Introduction}

At HERA, electrons ${ }^{1}$ of energy $27.5 \mathrm{GeV}$ collided head-on with protons of energy $920 \mathrm{GeV}$, resulting in an $e p$ centre-of-mass energy of $320 \mathrm{GeV}$. Such collisions were recorded by the H1 and ZEUS experiments. Neutral-current deep-inelastic scattering (NC DIS) is characterised by identifying the scattered electron in the detector. In this article, measurements of NC DIS reactions are reported with the additional condition of having signatures of a heavy (charm or beauty) quark in the detector. Two types of measurements are reported in the following. Leading order graphs corresponding to the two cases are shown in Figure 1.
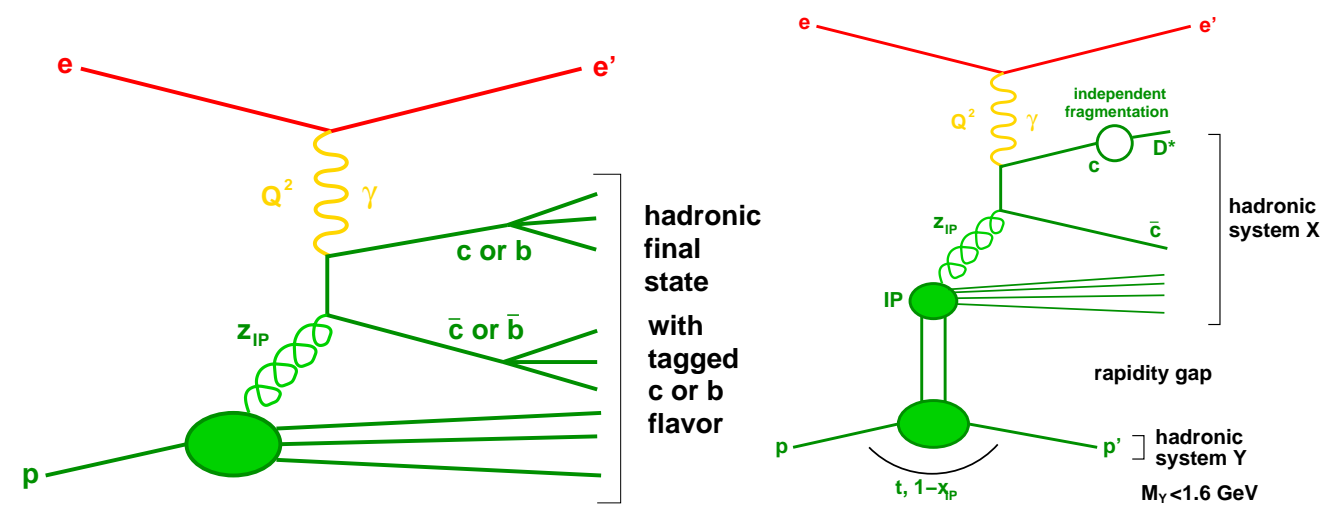

Figure 1: Leading order graphs illustrating the reactions discussed in this article. Left panel: production of charm or beauty in NC DIS. Right panel: production of $D^{*}(2010)$ mesons in diffractive NC DIS.

\section{Combination of HERA charm and beauty data}

Data on charm and beauty production previously published by the H1 and ZEUS collaborations are combined [1] as a function of the negative momentum transfer squared $Q^{2}$ and the Bjorken scaling variable $x$. While various techniques to tag charm or beauty events have been used in the original analyses, here the data are extrapolated to the full phase space at given $\left(x, Q^{2}\right)$ points. The reduced cross sections, given by

$$
\sigma_{\text {red }}^{q \bar{q}}=\frac{x Q^{4}}{2 \pi \alpha^{2}\left(1+(1-y)^{2}\right)} \frac{d^{2} \sigma^{q \bar{q}}}{d x d Q^{2}}
$$

are combined. The index $q \bar{q}=c \bar{c}$ or $b \bar{b}$ indicates the heavy quark flavour under investigation, $\alpha$ is the fine-structure constant and $y=Q^{2} /(s x)$ is the inelasticity. The gain in precision from combining data is illustrated in Figure 2. A total of 13 datasets are combined. Overall there are 52 charm data points and 27 beauty data points. In the combination there are 187 degrees of freedom and the minimum $\chi^{2}$ is 149 , taking into account statistical and systematic uncertainties. This demonstrates good consistency of the input datasets. The data are compared in Figure 3 to NLO theory predictions [2,3] and to predictions in approximate NNLO [4]. Also shown is the result of a simultaneous NLO QCD fit to this data and HERA inclusive DIS data, thus determining the proton 

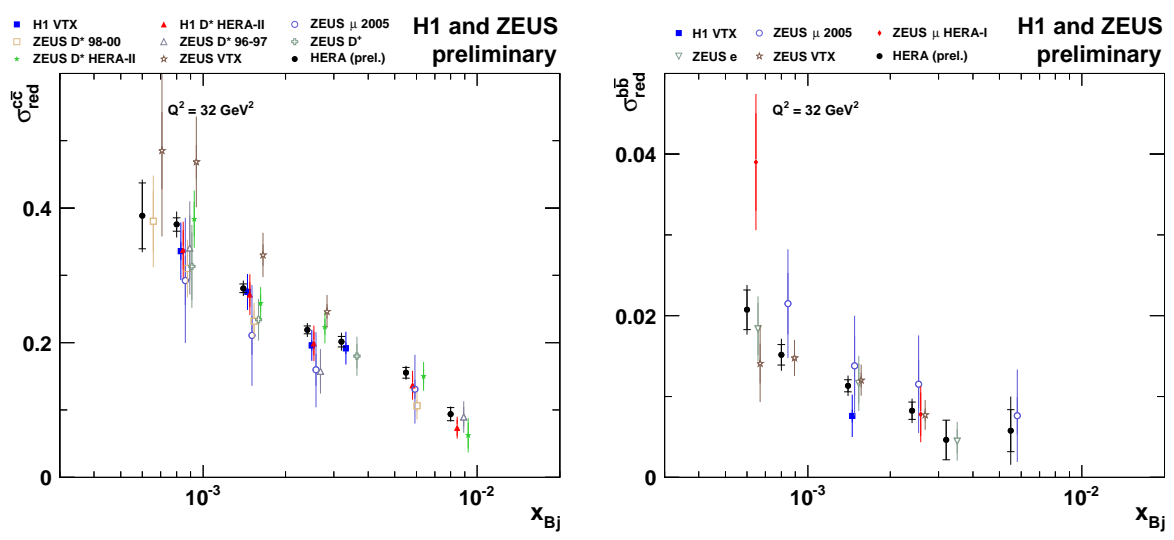

Figure 2: Comparison of the individual charm (left panel) and beauty (right panel) measurements of the reduced cross section for heavy quark production prior to the data combination. Shown are the data at $Q^{2}=32 \mathrm{GeV}^{2}$ as a function of $x$. The combined data are overlaid as solid dots. The individual measurements are offset horizontally in order to improve their visibility.
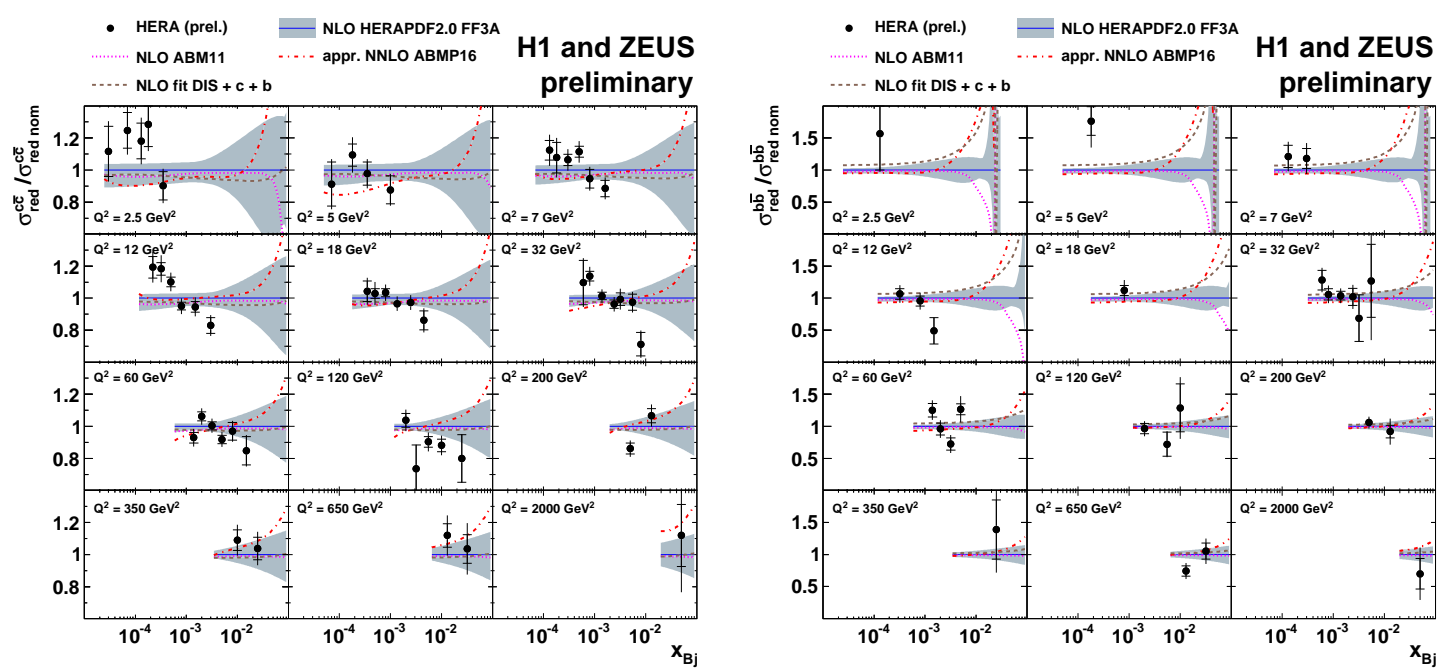

Figure 3: Comparison of charm (left panel) and beauty (right panel) measurements of the reduced cross section for heavy quark production to NLO QCD predictions and a fit of the data. Shown are ratios to the prediction based on HERAPDF2.0 FF3A [2].

parton density functions and the heavy quark masses together. There is a reasonable agreement between data and the predictions. However, at intermediate $Q^{2}$ near $12 \mathrm{GeV}^{2}$, the combined charm data seem to have a slope in $x$ which is different than predicted; the QCD fit to the data can not

\footnotetext{
${ }^{1}$ The generic term electron is used here to refer to both electrons and positrons.
} 
accommodate for this slope either. The charm and beauty running masses are extracted as

$$
\begin{aligned}
& m_{c}\left(m_{c}\right)=1209_{-41}^{+46}(\text { fit })_{-14}^{+62}(\text { model })_{-31}^{+7}(\text { param }) \mathrm{MeV} \text { and } \\
& m_{b}\left(m_{b}\right)=4049_{-109}^{+104}(\text { fit })_{-32}^{+90}(\text { model })_{-31}^{+1}(\text { param }) \mathrm{MeV} .
\end{aligned}
$$

The "fit" uncertainties encompass all experimental uncertainties. The "model" uncertainties originate from varying the scale definition or parameters such as the strong coupling. The "param" uncertainties arise from variations of the functional form of the parton densities or the starting scale of the evolution. The results compare well with the world averages, $m_{c}\left(m_{c}\right)=1270 \pm 30 \mathrm{MeV}$ and $m_{b}\left(m_{b}\right)=4180 \pm 30 \mathrm{MeV}[5]$.

\section{Measurement of $D^{*}(2010)$ meson production in diffractive NC DIS}

For this measurement by the $\mathrm{H} 1$ collaboration, $D^{*}(2010)$ mesons are identified in diffractive NC DIS. The diffractive signature is given by a large rapidity gap in the hadronic final state. The measurement phase space is defined by restrictions on the inclusive variables, $5<Q^{2}<100 \mathrm{GeV}^{2}$ and $0.02<y<0.65$, by the $D^{*}(2010)$ meson transverse momentum $p_{t, D^{*}}>1.5 \mathrm{GeV}$ and pseudorapidity $-1.5<\eta_{D^{*}}<1.5$ and by the large rapidity gap selection. That selection corresponds to requirements on the fractional longitudinal momentum loss of the incoming proton $x_{I P}<0.03$, the momentum transfer squared at the proton vertex $|t|<1 \mathrm{GeV}^{2}$ and the mass of the protondissociative system $M_{Y}<1.6 \mathrm{GeV}$. The variable $z_{I P}$, which corresponds to the longitudinal momentum of the $c \bar{c}$ system relative to the proton's longitudinal momentum loss, is also investigated. The measurements are confronted with NLO QCD calculations [6, 7], using also the diffractive factorisation theorem [8] and previously determined diffractive parton densities [9]. In order to compare directly to the $D^{*}(2010)$ data, the calculations are augmented by an independent fragmentation to $D^{*}(2010)$ mesons, using parameters determined previously in inclusive $D^{*}(2010)$ production [10]. Figure 4 shows the results, compared to the NLO QCD predictions. The data
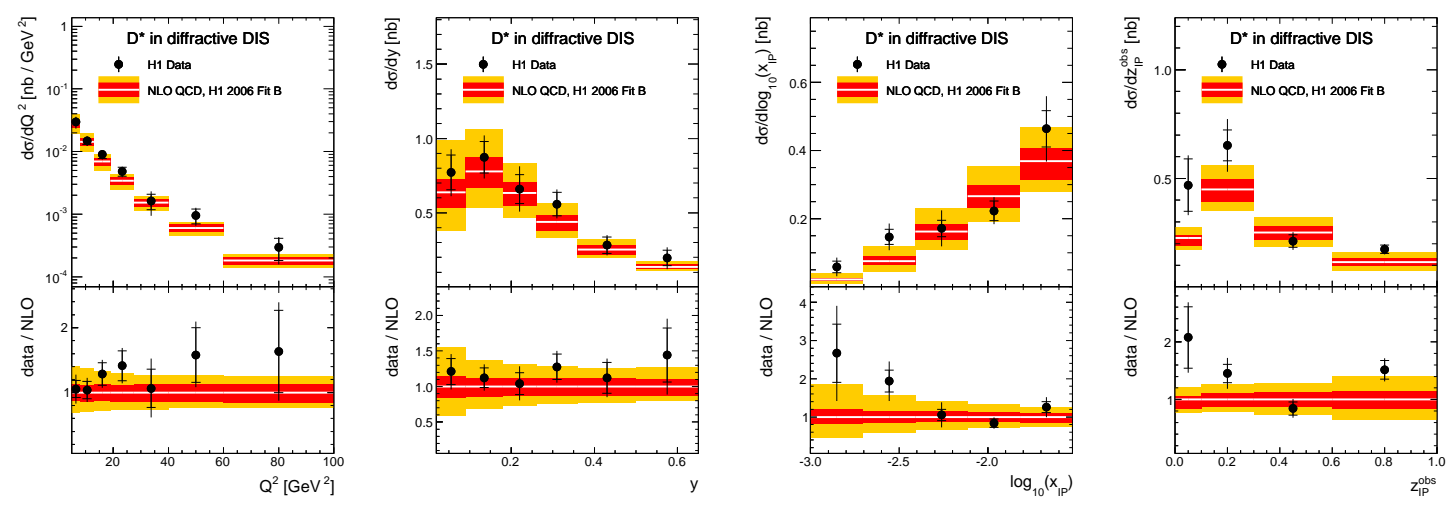

Figure 4: Measurements of $D^{*}(2010)$ meson production in diffractive DIS. Shown are single differential distributions (bin-integrated cross sections normalised to the bin width) as a function of the inclusive variables $Q^{2}, y$ and the diffractive variables $\log _{10} x_{I P}$ and $z_{I P}$. 
are well described by the NLO predictions, which however have sizeable uncertainties from scale variations and the limited knowledge of diffractive parton densities.

\section{Summary}

New measurements of heavy flavour production in NC DIS at HERA are presented. A combination of $\mathrm{H} 1$ and ZEUS data is targeting the best possible precision. NLO QCD calculations provide a reasonable description of the data. The running charm and beauty quark masses are extracted at NLO, in experimental precision competing with other world data. New measurements of $D^{*}(2010)$ production in diffractive NC DIS are an interesting tool to investigate QCD factorisation in diffraction. NLO calculations are able to describe these data, confirming the validity of the corresponding factorisation theorem in diffractive DIS. However, the comparisons suffer from rather large theory uncertainties driven by scale variations and the limited accuracy of diffractive parton densities.

\section{References}

[1] The H1 and ZEUS collaborations, H1prelim17-071 and ZEUS-prel-17-01, available at https://www.desy.de/h1zeus/combined_results/heavy_flavours

[2] H. Abramowicz et al. [H1 and ZEUS Collaborations], Eur. Phys. J. C 75 (2015) no.12, 580 doi:10.1140/epjc/s10052-015-3710-4 [arXiv:1506.06042].

[3] S. Alekhin, J. Blümlein and S. Moch, Phys. Rev. D 86 (2012) 054009 doi:10.1103/PhysRevD.86.054009 [arXiv:1202.2281].

[4] S. Alekhin, J. Blümlein, S. Moch and R. Placakyte, Phys. Rev. D 96 (2017) no.1, 014011 doi:10.1103/PhysRevD.96.014011 [arXiv:1701.05838].

[5] C. Patrignani et al. [Particle Data Group], Chin. Phys. C 40 (2016) no.10, 100001. doi:10.1088/1674-1137/40/10/100001

[6] B. W. Harris and J. Smith, Nucl. Phys. B 452 (1995) 109 doi:10.1016/0550-3213(95)00256-R [hep-ph/9503484].

[7] B. W. Harris and J. Smith, Phys. Rev. D 57 (1998) 2806 doi:10.1103/PhysRevD.57.2806 [hep-ph/9706334].

[8] J. C. Collins, Phys. Rev. D 57 (1998) 3051 doi:10.1103/PhysRevD.61.019902, 10.1103/PhysRevD.57.3051 [hep-ph/9709499]; erratum in Phys. Rev. D 61 (2000) 019902

[9] A. Aktas et al. [H1 Collaboration], Eur. Phys. J. C 48 (2006) 715 doi:10.1140/epjc/s10052-006-0035-3 [hep-ex/0606004].

[10] F. D. Aaron et al. [H1 Collaboration], Eur. Phys. J. C 59 (2009) 589 doi:10.1140/epjc/s10052-008-0792-2 [arXiv:0808.1003]. 